\title{
Variation of Daily Care Demand in Swiss General Hospitals: Longitudinal Study on Capacity Utilization, Patient Turnover and Clinical Complexity Levels
}

Narayan Sharma ${ }^{1}$, MSc; René Schwendimann ${ }^{1,2}, \mathrm{PhD}$; Olga Endrich ${ }^{3}, \mathrm{MD}$; Dietmar Ausserhofer ${ }^{1,4}$, PhD; Michael Simon $^{1,5}, \mathrm{PhD}$

\footnotetext{
${ }^{1}$ Institute of Nursing Science, Department of Public Health, Faculty of Medicine, University of Basel, Basel, Switzerland

${ }^{2}$ Patient Safety Office, University Hospital Basel, Basel, Switzerland

${ }^{3}$ Directorate of Medicine, Inselspital University Hospital Bern, Bern, Switzerland

${ }^{4}$ College of Health-Care Professions Claudiana, Bozen, Italy

${ }^{5}$ Nursing Research Unit, Inselspital University Hospital Bern, Bern, Switzerland
}

\section{Corresponding Author:}

Michael Simon, PhD

Institute of Nursing Science

Department Public Health

Faculty of Medicine, University of Basel

Bernoullistrasse 28

Basel, 4056

Switzerland

Phone: 41612070912

Fax: 41612070955

Email: m.simon@unibas.ch

\section{Abstract}

Background: Variations in hospitals' care demand relies not only on the patient volume but also on the disease severity. Understanding both daily severity and patient volume in hospitals could help to identify hospital pressure zones to improve hospital-capacity planning and policy-making.

Objective: This longitudinal study explored daily care demand dynamics in Swiss general hospitals for 3 measures: (1) capacity utilization, (2) patient turnover, and (3) patient clinical complexity level.

Methods: A retrospective population-based analysis was conducted with 1 year of routine data of 1.2 million inpatients from 102 Swiss general hospitals. Capacity utilization was measured as a percentage of the daily maximum number of inpatients. Patient turnover was measured as a percentage of the daily sum of admissions and discharges per hospital. Patient clinical complexity level was measured as the average daily patient disease severity per hospital from the clinical complexity algorithm.

Results: There was a pronounced variability of care demand in Swiss general hospitals. Among hospitals, the average daily capacity utilization ranged from $57.8 \%$ (95\% CI 57.3-58.4) to $87.7 \%$ (95\% CI 87.3-88.0), patient turnover ranged from $22.5 \%$ (95\% CI 22.1-22.8) to 34.5\% (95\% CI 34.3-34.7), and the mean patient clinical complexity level ranged from 1.26 (95\% CI 1.25-1.27) to 2.06 (95\% CI 2.05-2.07). Moreover, both within and between hospitals, all 3 measures varied distinctly between days of the year, between days of the week, between weekdays and weekends, and between seasons.

Conclusions: While admissions and discharges drive capacity utilization and patient turnover variation, disease severity of each patient drives patient clinical complexity level. Monitoring - and, if possible, anticipating-daily care demand fluctuations is key to managing hospital pressure zones. This study provides a pathway for identifying patients' daily exposure to strained hospital systems for a time-varying causal model.

(J Med Internet Res 2021;23(8):e27163) doi: $\underline{10.2196 / 27163}$

\section{KEYWORDS}

inpatient population; routine data; general hospitals; capacity utilization; clinical complexity; patient data; hospital system; complexity algorithm 


\section{Introduction}

Hospitals are constantly challenged by changing patient care demands. If this outweighs available resources, it can affect the quality of care and patient safety [1]. Demand factors include daily patient volume, turnover, and clinical complexity of patients requiring diagnoses and therapies [1,2]. Responding to variations in any of these factors, hospitals adjust their resource supplies (eg, by changing shift-level staffing or resources for each day, between workdays and weekends, for different seasons, and throughout the year) $[3,4]$.

Capacity utilization, which is based on the number of beds occupied vs those available [5], offers one perspective to view hospital care demand $[2,6,7]$. Over recent decades, most health care systems' capacity utilization has increased, while total numbers of available beds have decreased [3,8]. This trend mainly reflects policies to reduce health care costs and to increase efficiency (eg, by the use of diagnostic-related groups [DRGs]) $[8,9]$.

If capacity utilization is too high (eg, above $80 \%$ or $85 \%$ ), it might overburden health care systems and their workforces $[10,11]$, possibly leading to adverse patient outcomes such as infections or even death $[5,12,13]$. Capacity utilization is high-exceeding 90\% — in Canada, Israel, and Ireland, followed by the United Kingdom, Norway, and Switzerland, all of which report figures above $80 \%$ [14]. Within a country, capacity utilization also varies between hospital types, geographic regions, and populations served [6,15]. In Switzerland, the most recent annual capacity utilization figures for acute care hospitals were between $70 \%$ and $82 \%[16,17]$.

As noted above, care demand also relies on patient turnover and patient complexity [18]. Patient turnover refers to the admission and the discharge or transfer of patients between units or hospitals [19], requiring resources [20]. "Census variability," "churn," or "environmental turbulence" cover the same or similar concepts [21]. Patient complexity refers to the severity or complexity of each patient's clinical needs. For instance, patients admitted to the intensive care unit generally require more resources than those in a general ward, representing a resource-intensive caseload.

Disease severity is commonly measured via the Charlson-Elixhauser comorbidity or case-mix index; however, this does not include all relevant clinical conditions or morbidities, and its interpretation is commonly influenced by reimbursement policies or the cost of medication and treatment [22-24]. One alternative measure is the patient clinical complexity level. As part of the German DRG system, the patient clinical complexity level reflects upon not only complications and comorbidities but also their levels of clinical severity on a 5 -point scale (ie, $0=$ no, $1=$ mild, $2=$ moderate, $3=$ severe, and 4=very severe clinical complexity) [25]. A complex algorithm, depending on primary and secondary diagnoses and estimated severities, allows determination of the cumulative effect of the diagnoses per treatment episode $[22,26,27]$. A higher patient clinical complexity level indicates a more complex and resource-intensive caseload.
As capacity utilization, patient turnover, and patient clinical complexity level all offer necessary perspectives on hospital care demand, all 3 are vital for optimal resource allocation [28]. All 3 are also connected; for instance, complex patients usually stay longer in hospitals, increasing capacity utilization. Furthermore, with each transfer or referral to another hospital, additional load is created, as patients need to be assessed at admission or be prepared for discharge [21]. Understanding these factors' daily variation is a vital step toward optimizing health care structures and processes [29]. Regarding the daily variability of care demand, analysis of long-term data can help to anticipate when staffs or supplies will be depleted or strained, thereby, indicating when and where to allocate resources. Particularly, the traditional measures of capacity utilization (eg, midnight count $[29,30])$ and patient turnover (eg, the inverse of the length of hospital stay [31,32]) may not convey the dynamic nature of actual daytime hospital care demands [33]; certainly, neither incorporates patient complexity and severity.

Additionally, the valid, highly granular longitudinal (daily or weekly) measurement of care demand offers a precise and in-depth view of how care needs fluctuate and evolve. As capacity utilization, patient turnover, and patient clinical complexity level are time-sensitive variables, the 3 together offer great potential to accurately represent daily care demand dynamics. Such information should enable health care managers to anticipate capacity needs to accommodate patients during a typical weekday, weekend, or seasonal peak [3].

Therefore, this study aimed to describe the daily care demand in general hospitals from a longitudinal perspective, specifically, the daily peaks and variations during weeks (ie, weekends vs weekdays), as well as seasons. This study describes the daily variability of (1) capacity utilization, (2) patient turnover, and (3) patient clinical complexity levels of Swiss general hospitals' inpatient populations.

\section{Methods}

\section{Study Design, Setting, and Population}

This is a retrospective, population-based analysis using 1 year of hospital data extracted from the 6-year (2012-2017) dataset obtained from the Swiss Federal Statistics Office (FSO). Based on a data protection contract (as stipulated by article 22 of the Swiss Federal Act on Data Protection), the FSO provided anonymized data from all Swiss hospital inpatients hospitalized over the study period. The data covered general as well as specialized care facilities such as pediatric, gynecological, psychiatric, and rehabilitative hospitals [34]. The FSO divides general hospitals into 5 classifications: university hospitals, tertiary care hospitals, large basic hospitals, medium basic hospitals, and small basic hospitals. Classification is based on the number of cases treated per year and a weighted sum of service points (based on a combination of the number of hospital units and the levels of care delivered) assigned by the Swiss Medical Association [17,34]. For instance, based on the Swiss Medical Association classification, a university hospital requires the weighted sum of service points to be $>100$ units and $>30,000$ cases per year [34]. For this study, we included a 1-year patient population dataset from general hospitals to limit interhospital 
heterogeneity. Due to the Swiss Data Protection Act's stipulations regarding patient anonymity, we were unable to trace patients across years. An overview of the process of selecting inpatient cases for analysis is included in the flow diagram in Figure 1.

Figure 1. Flow diagram of inpatient cases for the analysis.

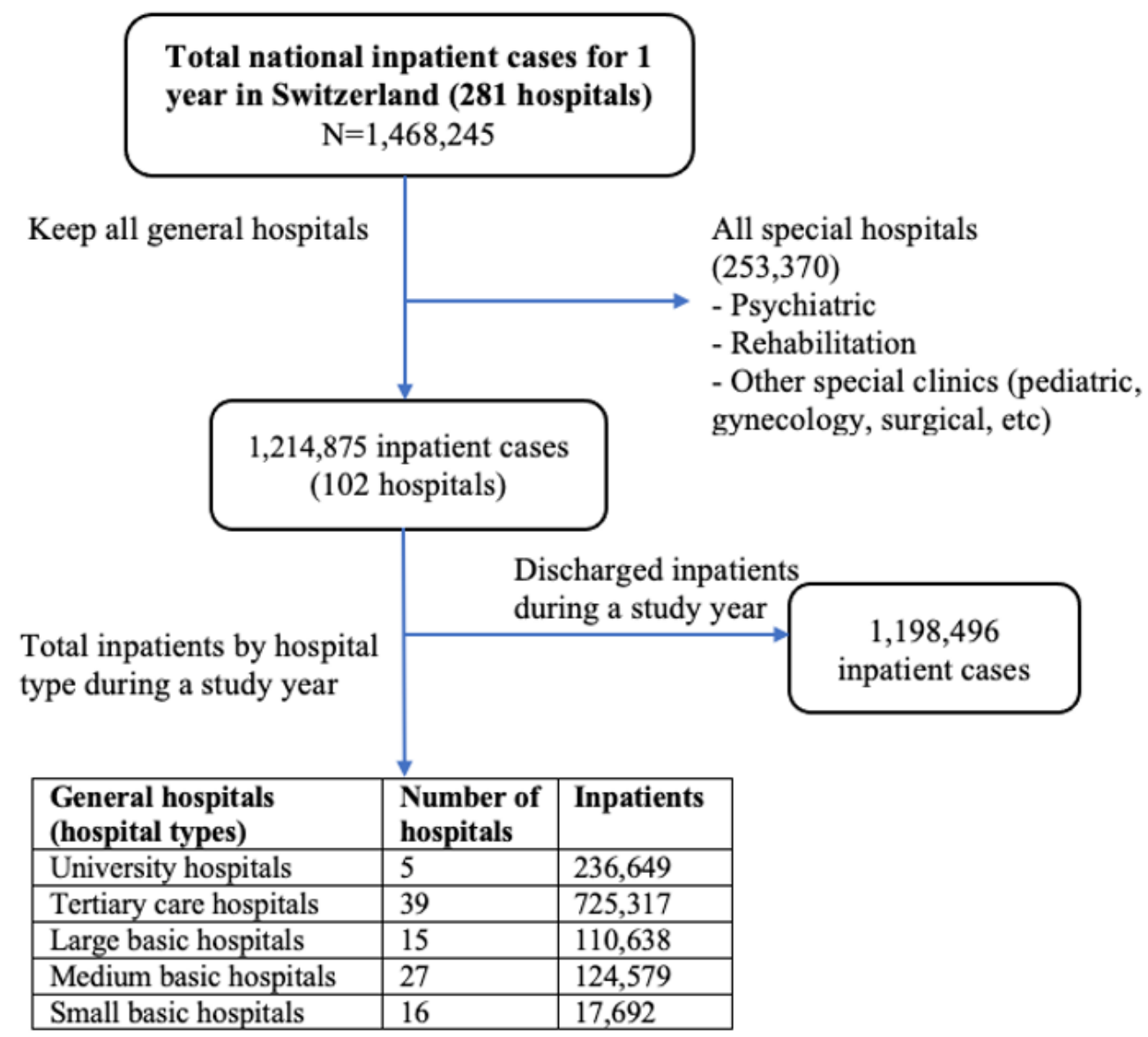

\section{Dataset and Study Variables}

We extracted 1-year datasets, including (1) all inpatients discharged during each study year, regardless of admission date, and (2) all inpatients admitted during each study year, regardless of discharge date. From the full FSO hospital dataset, we also extracted data for all relevant routine administrative and clinical variables for the study period (Multimedia Appendix 1).

\section{Statistical Analyses}

All statistical analyses were conducted using R, version 3.6.3 for Mac OS [35]. The following statistical packages were used: (1) dplyr [36] and tidyr [37] for data preparation; (2) lubridate [38] and stringr [39] for handling time and date; and (3) ggplot2 [40], patchwork [41], and scales [42] for plotting.

\section{Descriptive Overview}

For each hospital type, in addition to the number of hospitals, the total numbers of patients, admissions, and discharges were recorded by gender in frequencies and percentages. Length of stay (LOS), in days, was calculated for all patients by subtracting each individual's admission date from their discharge date. The results were aggregated at the level of the hospital type and presented as mean $(95 \% \mathrm{CI})$ and median (IQR), for each type.

\section{Capacity Utilization Measure}

For each hospital, each study day's capacity utilization was calculated as a percentage, using that hospital's highest recorded daily bed occupancy for that year as the denominator [5,9]. Each patient's admission and discharge dates were used to calculate the number of patients present each day in each hospital. The capacity utilization of a day in a given hospital included all patients admitted or discharged on that day and the number who were present the previous day and not discharged or deceased. As noted above, patients admitted before the study year (eg, in December of the previous year) were included in the study until their discharge. Patients not discharged during the study year were included until the end of the study year. Total capacity utilization was calculated for each hospital $(n=102)$ for 365 days (with a total of 37,230 time points).

Furthermore, daily percentages of capacity utilization were summarized by hospital type, along with means (95\% CIs), SDs, and minimum-maximum values. To visualize daily variations in capacity utilization, smoothed lines were plotted, with the $95 \%$ CIs around the mean, for each hospital type. For each hospital type, we also plotted graphs to show variation by day of the week. Finally, for each hospital type, weekday vs weekend variations were plotted for each week of the year. 
There was no dot plotted for weekdays of the first week, as the first day of this study year was a weekend (Sunday).

\section{Patient Turnover Measure}

As calculated for capacity utilization, daily patient turnover was calculated for each hospital and aggregated by hospital type. The patient turnover rate was calculated as absolute counts of admissions, discharges, and deceased patients for a particular day divided by the total number of patients for that day per hospital [21]. As opposed to using the inverse of the average LOS method, this approach has the advantage of adequately representing the volume of activity either for entire days or short hospital stays as contributors to increasing patient throughput $[19,21,43]$. The percentage of patient turnover per day was calculated and further summarized by hospital type as mean (95\% CI), SD, and minimum-maximum figures. To display daily variations in patient turnover, similar displays were plotted for them as for capacity utilization.

\section{Patient Clinical Complexity Level}

Our patient clinical complexity level data covered only patients discharged during the study year (as the International Classification of Diseases [ICD]-10 codes were not available for patients until they were discharged), and Swiss DRG version 6 was applied for that study year [44]. The patient clinical complexity level calculation was based on a complex algorithm, providing clinical complexity and comorbidity level values (ranging from 0-4) for all possible primary and secondary diagnoses per patient case [26]. Developed as part of the complexity and comorbidity level Refinement Project in Australia [26,27,45], this algorithm was applied to determine each patient's final patient clinical complexity level. To facilitate this process, we used the grouping system provided by Swiss DRG AG [44].

We began by organizing our data input into a readable format via the grouping system. We chose the "SwissDRG Batchgrouper Format 2017" short input format, which provides an anonymous case identifier, plus the patient's age, sex, admission and discharge date, LOS, primary and secondary diagnoses, and treatment procedure codes. "DRG Output format for SwissDRG" results were then obtained, including patient clinical complexity level values for each case. Individual patient clinical complexity level values were further transformed to average daily patient clinical complexity level values per hospital, using each patient's admission and discharge dates (ie, each case's patient clinical complexity level value is applied to each day for that hospital until discharge). Daily patient clinical complexity level values were further summarized by hospital type as means (95\% CIs), SDs, and minimum-maximum figures. As for the other 2 measures, similar displays were plotted to display variation in daily patient clinical complexity levels.

\section{Results}

\section{Descriptive Overview}

During the study year, $1,214,875$ inpatients stayed in the 102 Swiss general hospitals, of which 16,379 cases $(1.35 \%)$ continued to the following year. Of the $1,214,875$ inpatients, the 5 university hospitals covered $19.50 \%(n=236,649)$, the 39 tertiary care hospitals covered $59.70 \%(\mathrm{n}=725,317)$, and the 58 basic hospitals covered $20.81 \%(n=252,909)$ of the patient population. Overall, of the $1,214,875$ inpatients, there were approximately $6.87 \%(n=91,078)$ more female than male patients. The average patient LOS across all general hospitals was 6.43 (95\% CI 6.40-6.46) days; the median LOS was 3.7 (IQR 2.0-7.0) days. The general characteristics of the study population by hospital type are presented in Multimedia Appendix 1.

\section{Variation of Daily Capacity Utilization}

Average daily capacity utilization ranged from 527-2340 patients in university hospitals, 87-1099 patients in tertiary care hospitals, 16-304 patients in large basic hospitals, 7-179 patients in medium basic hospitals, and 1-93 patients in small basic hospitals. Notably, 3 small basic hospitals had average daily capacity utilization numbers below 10 patients.

Across the study period, the average daily capacity utilization was highest in university hospitals and the lowest in small basic hospitals (Table 1). However, the range (ie, the variation between the lowest and the highest daily capacity utilizations for a study year) was almost $98 \%$ (eg, 1.7-100.0) for small basic hospitals, $92 \%$ for medium basic hospitals, $87 \%$ for large basic hospitals, $73 \%$ for tertiary care hospitals, and $44 \%$ for university hospitals. 
Table 1. Daily capacity utilization, patient turnover, and patient clinical complexity level per hospital, by hospital type, from the 1-year patient population.

\begin{tabular}{|c|c|c|c|c|c|c|c|c|c|}
\hline \multirow[t]{2}{*}{ Hospital type $\left(\right.$ median $\left.^{\mathrm{a}}\right)$} & \multicolumn{3}{|c|}{ Capacity utilization (\%) } & \multicolumn{3}{|c|}{ Patient turnover ${ }^{\mathrm{b}}(\%)$} & \multicolumn{3}{|c|}{ Patient clinical complexity level $\left(0-4^{c}\right)$} \\
\hline & Mean (SD) & $95 \% \mathrm{CI}$ & Min-max ${ }^{\mathrm{d}}$ & Mean (SD) & $95 \% \mathrm{CI}$ & Min-max & Mean (SD) & $95 \% \mathrm{CI}$ & Min-max \\
\hline University hospitals (988) & $87.7(7.7)$ & $87.3-88.0$ & $55.8-100.0$ & $22.5(7.6)$ & $22.1-22.8$ & $5.7-38.7$ & $2.06(0.2)$ & $2.05-2.07$ & $0.81-2.57$ \\
\hline $\begin{array}{l}\text { Tertiary care hospitals } \\
\text { (298) }\end{array}$ & $78.7(10.2)$ & $78.5-78.9$ & $27.3-100.0$ & $28.8(7.5)$ & $28.6-28.9$ & $2.7-54.6$ & $1.78(0.3)$ & $1.78-1.79$ & $0.42-2.75$ \\
\hline Large basic hospitals (120) & $71.3(13.4)$ & $70.9-71.6$ & $13.1-100.0$ & $32.6(9.2)$ & 32.4-32.9 & $0.0-75.4$ & $1.46(0.4)$ & $1.45-1.47$ & $0.09-2.50$ \\
\hline $\begin{array}{l}\text { Medium basic hospitals } \\
\text { (71) }\end{array}$ & $65.3(15.2)$ & $65.0-65.6$ & $5.9-100.0$ & $34.5(10.7)$ & $34.3-34.7$ & $0.0-109.1$ & $1.26(0.6)$ & $1.25-1.27$ & $0.00-2.93$ \\
\hline Small basic hospitals (19) & $57.8(22.2)$ & $57.3-58.4$ & $1.7-100.0$ & $24.3(22.0)$ & $23.8-24.9$ & $0.0-200.0$ & $1.65(0.8)$ & $1.63-1.67$ & $0.00-4.00$ \\
\hline
\end{tabular}

${ }^{\mathrm{a}}$ Median number of beds used per day per hospital, by hospital type.

${ }^{b}$ Patient turnover is the percentage of total patients admitted and discharged in a day.

${ }^{\mathrm{c}} 0=$ No clinical complexity, $1=$ mild clinical complexity, $2=$ severe clinical complexity, and $4=$ very severe clinical complexity.

d Min-max: Minimum-maximum value on hospital level within hospital type.

As indicated by the smooth curves and line charts by hospital utilization was lower throughout the summer months categories, university hospitals' daily capacity utilizations were (June-August; Figure 2).

high throughout the year. Among all hospital types, capacity 
Figure 2. Daily capacity utilization of Swiss general hospital types for 1 year (smooth curve with mean between CIs and line chart).
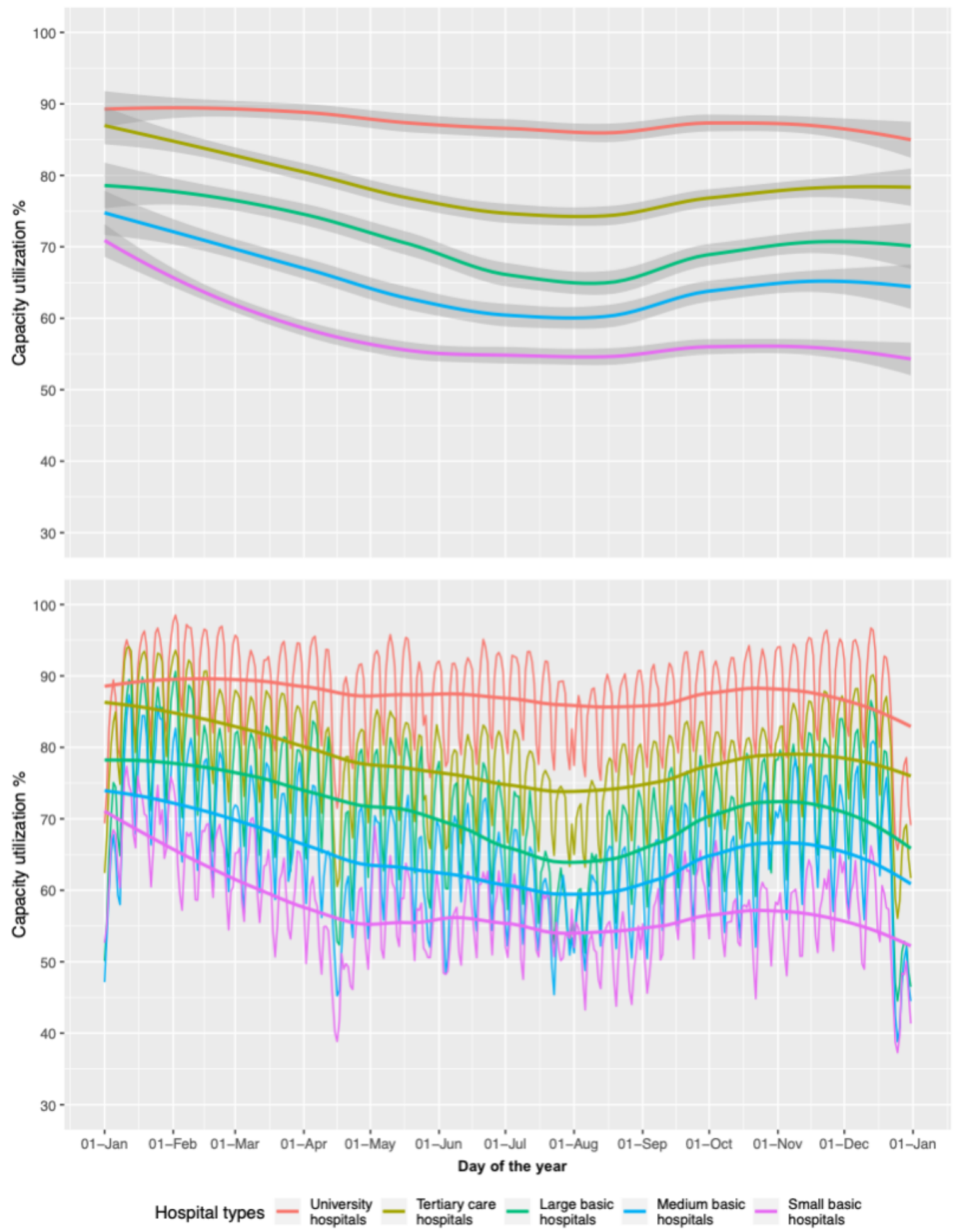

There was a gradual increase in capacity utilization through the all hospital types. There was roughly a $10 \%$ difference in early days of the week (Mondays-Wednesdays; Figure 3), capacity utilization during weekdays than on the weekend, in followed by a gradual decrease from Fridays to Sundays, across all hospital types (Table 2). 
Figure 3. Capacity utilization of Swiss general hospital types with mean between CI by day of the week.

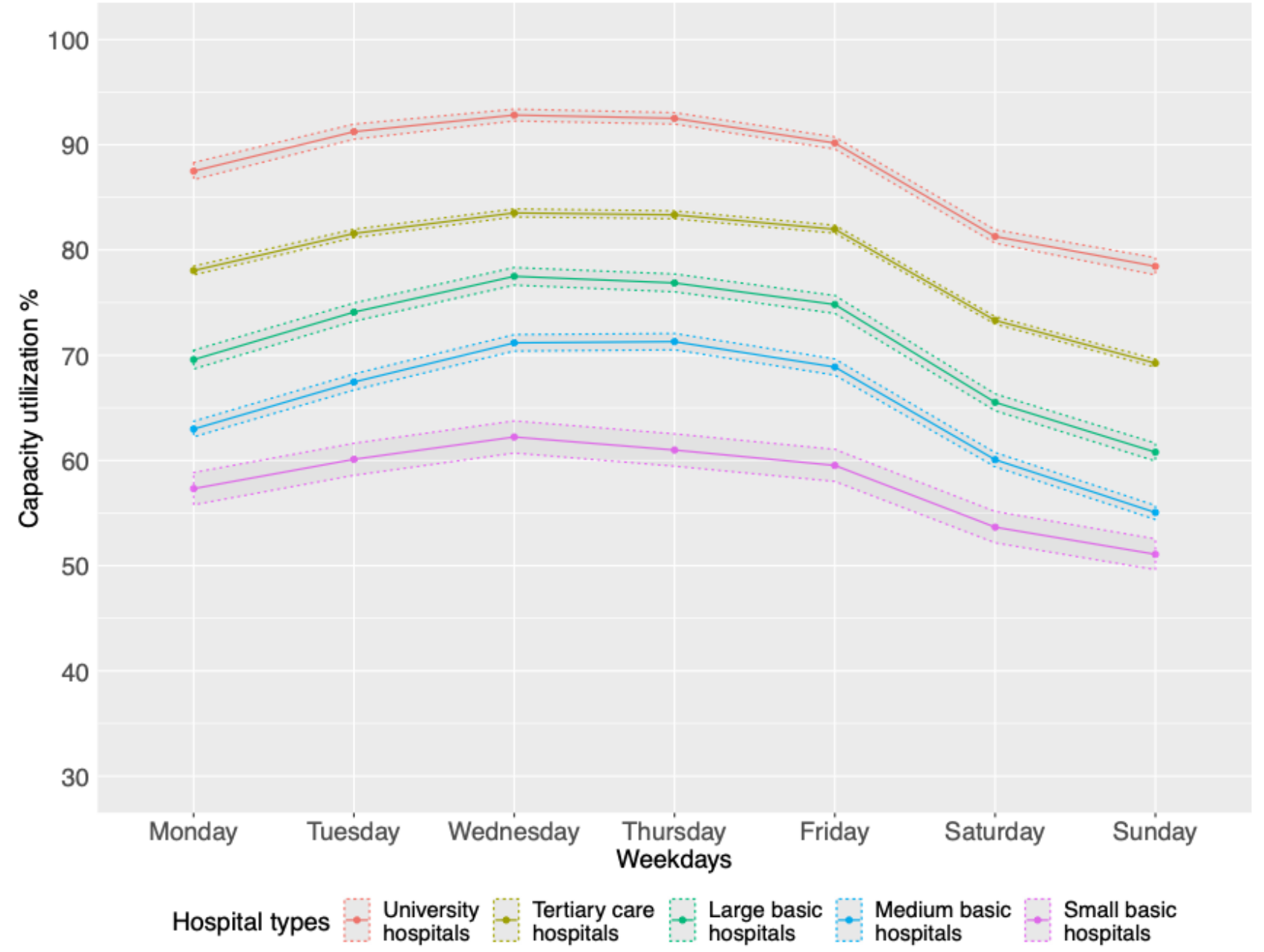


Table 2. Daily capacity utilization, patient turnover, and patient clinical complexity level by weekday vs weekend from the 1-year patient population.

\begin{tabular}{|c|c|c|c|c|c|c|c|c|c|}
\hline \multirow[t]{2}{*}{ Day by hospital type } & \multicolumn{3}{|c|}{ Capacity utilization (\%) } & \multicolumn{3}{|c|}{ Patient turnover $(\%)$} & \multicolumn{3}{|c|}{ Patient clinical complexity level $\left(0-4^{\mathrm{a}}\right)$} \\
\hline & Mean (SD) & $95 \% \mathrm{CI}$ & Min-max ${ }^{b}$ & Mean (SD) & $95 \% \mathrm{CI}$ & Min-max & Mean (SD) & $95 \% \mathrm{CI}$ & Min-max \\
\hline \multicolumn{10}{|l|}{ University hospitals } \\
\hline Weekday & $90.8(3.8)$ & $89.8-91.9$ & $72.8-96.2$ & $25.5(1.4)$ & $25.1-25.9$ & $16.6-26.6$ & $2.04(0.21)$ & $2.03-2.05$ & $1.22-2.57$ \\
\hline Weekend & $79.8(3.0)$ & 78.9-80.6 & $69.1-85.0$ & $14.8(0.8)$ & $14.6-15.1$ & $10.5-17.6$ & $2.1(0.27)$ & $2.08-2.13$ & $0.81-2.55$ \\
\hline \multicolumn{10}{|c|}{ Tertiary care hospitals } \\
\hline Weekday & $81.7(5.6)$ & $80.1-83.3$ & $63.5-92.1$ & $31.9(1.6)$ & $31.4-32.3$ & $22.2-33.2$ & $1.76(0.28)$ & $1.75-1.77$ & $0.84-2.69$ \\
\hline Weekend & $71.2(4.5)$ & $69.9-72.4$ & $61.6-80.9$ & $21.1(1.1)$ & $20.8-21.4$ & $14.4-24.3$ & $1.83(0.30)$ & $1.82-1.84$ & $0.42-2.75$ \\
\hline \multicolumn{10}{|l|}{ Large basic hospitals } \\
\hline Weekday & $74.6(7.4)$ & $72.5-76.6$ & $49.6-86.8$ & $35.9(1.9)$ & $35.4-36.4$ & $25.2-37.9$ & $1.44(0.38)$ & $1.42-1.45$ & $0.14-2.25$ \\
\hline Weekend & $63.0(5.9)$ & 61.4-64.6 & $46.5-74.4$ & $24.4(1.6)$ & $23.9-24.8$ & $16.7-29.5$ & $1.52(0.38)$ & $1.5-1.54$ & $0.09-2.5$ \\
\hline \multicolumn{10}{|c|}{ Medium basic hospitals } \\
\hline Weekday & $68.4(7.0)$ & $66.4-70.3$ & $45.8-82.0$ & $37.1(1.3)$ & $36.8-37.5$ & $31.2-39.3$ & $1.24(0.54)$ & $1.22-1.25$ & $0.05-2.79$ \\
\hline Weekend & $57.6(5.5)$ & $56.1-59.1$ & $44.5-70.0$ & $27.8(1.7)$ & $27.4-28.3$ & $24.0-34.6$ & $1.31(0.57)$ & $1.29-1.33$ & $0.00-2.93$ \\
\hline \multicolumn{10}{|l|}{ Small basic hospitals } \\
\hline Weekday & $60.0(5.9)$ & $58.3-61.6$ & $44.6-74.1$ & $27.0(2.4)$ & $26.3-27.6$ & $22.2-31.7$ & $1.63(0.80)$ & $1.6-1.65$ & $0.00-4.00$ \\
\hline Weekend & $52.2(5.3)$ & $50.8-53.7$ & $41.4-65.7$ & $18.0(4.0)$ & $16.9-19.1$ & $11.3-28.8$ & $1.69(0.81)$ & $1.65-1.73$ & $0.00-4.00$ \\
\hline
\end{tabular}

${ }^{\mathrm{a}} 0=$ No clinical complexity, $1=$ mild clinical complexity, $2=$ severe clinical complexity, and $4=$ very severe clinical complexity.

${ }^{\mathrm{b}}$ Min-max: Minimum-maximum.

Comparing weekdays with weekends, variations in capacity utilization for each week are shown in Figure 4. With very few exceptions (eg, the 2nd, the 17th, and the final week of the year), the weekly capacity utilization for weekdays was higher than for weekends. These weekly graphs also show lower capacity utilization during the summer months (weeks 20-35). 
Figure 4. Capacity utilization of Swiss general hospital types with mean between CI by weekday vs weekend for 1 year.
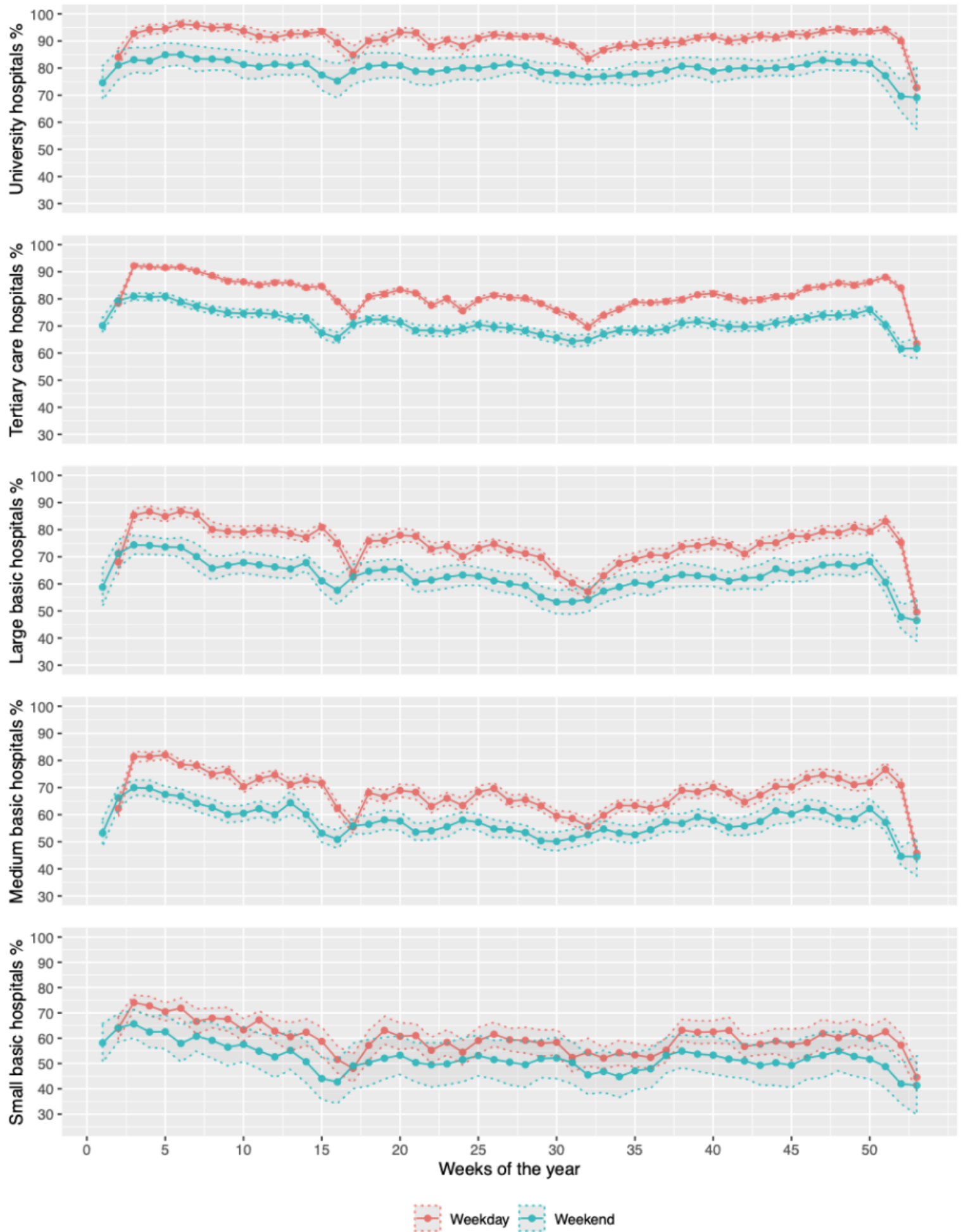

\section{Variation of Daily Patient Turnover}

Throughout the year, daily patient turnover ranged from 72-468 patients for university hospitals, 5-318 patients for tertiary care hospitals, 0-91 patients for large basic hospitals, 0-78 patients for medium basic hospitals, and 0-33 patients for small basic hospitals. The minimum value of 0 indicates that some hospitals saw neither admissions nor discharges on some days.
During the study year, the mean daily patient turnover percentage was highest in medium-sized basic hospitals and lowest in university hospitals (Table 1). The difference in daily patient turnover range was highest for small basic hospitals, decreasing with each increase in hospital size class. And, as illustrated in the smooth and line chart, daily turnover varied the least in university hospitals and the most in medium basic hospitals (Multimedia Appendix 2). 
Exploring the frequency of change in patient movement by day of the week (Multimedia Appendix 2), we found that turnover was highest on Mondays and lowest during weekends. Differences in patient turnover during weekdays and weekends across the 5 hospital types are shown in Table 2. Across the 5 hospital types, daily patient turnover was almost $10 \%$ higher on weekdays than on weekends.

The variation of patient turnover for each day of the week and for weekdays vs weekends is shown in Multimedia Appendix 2. In small basic hospitals, with few exceptions (eg, the 31st and 52nd weeks), the mean daily patient turnover was higher for weekdays than for weekends, for all hospital types. Overall patient turnover was lower during the holiday seasons (ie, weeks 17-18 and the final 2 weeks of the year).

\section{Variation of Daily Patient Clinical Complexity Level}

Overall, of the total discharged patients $(1,198,496), 10.97 \%$ $(\mathrm{n}=131,442)$ of patients had severe clinical complexity (patient clinical complexity level 4), while roughly $60.97 \%(n=730,893)$ had no clinical complexity (patient clinical complexity level 0; Table 3).

Table 3. Patient clinical complexity level by hospital type $(\mathrm{N}=1,198,496)$.

\begin{tabular}{|c|c|c|c|c|c|}
\hline \multirow[t]{2}{*}{ Hospital type $(\mathrm{N})$} & \multicolumn{5}{|c|}{ Patient clinical complexity level (0-4), n (\%) } \\
\hline & $\begin{array}{l}\text { 0: No clinical com- } \\
\text { plexity, } 730,893, \\
(60.98)\end{array}$ & $\begin{array}{l}\text { 1: Mild clinical com- } \\
\text { plexity, } 17,579(1.47)\end{array}$ & $\begin{array}{l}\text { 2: Moderate clinical } \\
\text { complexity, 136,453 } \\
(11.39)\end{array}$ & $\begin{array}{l}\text { 3: Severe clinical } \\
\text { complexity, } 182,129 \\
(15.20)\end{array}$ & $\begin{array}{l}\text { 4: Very severe clinical } \\
\text { complexity, } 131,442 \\
(10.97)\end{array}$ \\
\hline $\begin{array}{l}\text { University hospitals } \\
(\mathrm{n}=232,127)\end{array}$ & $122,567(52.8)$ & $3281(1.4)$ & $28,812(12.4)$ & $41,159(17.7)$ & $36,308(15.6)$ \\
\hline $\begin{array}{l}\text { Tertiary care hospitals } \\
(\mathrm{n}=715,809)\end{array}$ & $433,514(60.6)$ & $11,003(1.5)$ & $83,374(11.6)$ & $110,756(15.5)$ & $77,162(10.8)$ \\
\hline $\begin{array}{l}\text { Large basic hospitals } \\
(\mathrm{n}=109,572)\end{array}$ & $73,800(67.4)$ & $1397(1.3)$ & $10,801(9.9)$ & $14,590(13.3)$ & $8984(8.2)$ \\
\hline $\begin{array}{l}\text { Medium basic hospitals } \\
(\mathrm{n}=123,530)\end{array}$ & $90,284(73.1)$ & $1679(1.4)$ & $11,237(9.1)$ & $12,865(10.4)$ & $7465(6.0)$ \\
\hline $\begin{array}{l}\text { Small basic hospitals } \\
(\mathrm{n}=17,458)\end{array}$ & $10,728(61.5)$ & $219(1.3)$ & $2229(12.8)$ & $2759(15.8)$ & $1523(8.7)$ \\
\hline
\end{tabular}

Throughout the year, mean daily patient clinical complexity level varied across the 5 hospital types. It was highest in university hospitals $(2.06,95 \%$ CI $2.05-2.07)$ and lowest in medium basic hospitals (1.26, 95\% CI 1.25-1.27; Table 1). This is depicted in the smooth and line chart for the 5 general hospital types (Multimedia Appendix 3). Mean patient clinical complexity level gradually decreased from Monday until midweek but remained highest during the weekend-the opposite of the usual pattern of capacity utilization and patient turnover explored by the days of the week (Multimedia Appendix 3).

Weekday and weekend differences in patient clinical complexity level for the 5 general hospital types are shown in Table 2. Across all hospital types, patient clinical complexity level was almost 0.07 points higher during the weekend than on weekdays. During weekdays, university hospitals' average daily patient clinical complexity level was 2.04 (95\% CI 2.03-2.05); during the weekend, it was 2.1 (95\% CI 2.08-2.13). Weekday vs weekend patient clinical complexity level variation over 1 year is shown in Multimedia Appendix 3. Except for a small number of weeks (eg, the 7 th and the 31 st weeks in small basic hospitals), across all hospital types, the patient clinical complexity level for weekends was higher than for weekdays. Moreover, except for small basic hospitals, patient clinical complexity level dropped in December. This was partly because patient clinical complexity levels could only be calculated for patients discharged during the study year (ie, ICD diagnostic codes were unavailable for patients not discharged during the year, and anonymity considerations made it impossible to track patients across years). However, mean values both for patient clinical complexity level and for LOS were also lower for patients discharged in November and December, with higher patient clinical complexity level values assigned to patients who remained in the hospital across the years' end (Multimedia Appendix 4).

\section{Discussion}

\section{Principal Findings}

We examined 1 year of routine patient data from all 102 general hospitals in Switzerland. Average daily capacity utilization varied widely, from $57.8 \%$ in small basic hospitals to $87.7 \%$ in university hospitals. However, patient turnover was highest, at $34.5 \%$, in medium basic hospitals and lowest, at $22.5 \%$, in university hospitals. Moreover, average daily patient clinical complexity level was highest in university hospitals, at 2.06, and lowest in medium basic hospitals, at 1.26. Surprisingly, in small basic hospitals, patient turnover was lower than in tertiary hospitals or either of the 2 other basic hospital types, both of which also had higher mean patient clinical complexity levels throughout the year. Another interesting finding was that the average daily patient clinical complexity level was highest on weekends. Additionally, all hospital types showed distinct weekday, weekend, and seasonal effects regarding capacity utilization, patient turnover, and patient clinical complexity level.

Concerns have been raised that capacity utilization alone does not explain hospitals' total care demand $[6,18]$. Therefore, we viewed this alongside daily volumes of admitted and discharged 
patients and complexity [18]. This study explored all 3 measures, showing that capacity utilizations and patient clinical complexity levels were highest but patient turnovers were lowest in university hospitals. Even with a large proportion of inpatients in tertiary care institutions, university hospitals generally operate at close to full capacity and with the most complex patient cases. Thus, more care resources need to be allocated to university hospitals [3]. On the other hand, in small basic hospitals, where capacity utilization and patient turnover were relatively low, patient clinical complexity level was above those of the other basic hospitals. This indicates that complex cases are still treated in small basic hospitals, possibly, due to geographic proximity, which may also relate to older patients' preference for them: across all hospital types, these hospitals have the highest mean patient age. In light of these small basic hospitals' continued relevance (as they still treat complex cases), they may warrant greater resource allocation.

We measured daily demand for Swiss general hospital care longitudinally for 1 year. As it has also been observed in other studies regarding days of the week, Saturdays and Sundays had the lowest capacity utilization and patient turnover $[3,46]$. Moreover, we observed that patient clinical complexity level was highest during weekends, possibly, because more complex patients remain in the hospital through the weekend. Comparing weekly demand throughout the year, a clear distinction between weekends and weekdays was shown, with the highest variability occurring in small basic hospitals, possibly indicating suboptimal patient flow. Concurrently, seasonal variations were also seen. Capacity utilization was mainly highest in the winter and relatively low in the summer months, whereas patient turnover was constant throughout the year, dropping off toward the end of the year. However, patient clinical complexity level remained quite constant, with a slight drop during the summer months and a marked reduction during December. These changes tended to correspond with holidays, possibly, including higher patient discharge rates and fewer admissions before the holidays and at the end of the year. The capture of daily patient complexity during the end of the year was also reduced because ICD-10 codes were unavailable for patients who were admitted but not discharged during the study year. Furthermore, patients with higher patient clinical complexity levels were more likely to have longer LOSs, particularly, across the Christmas and end-of-year period.

\section{Potential Implications}

Based on capacity utilization, patient turnover, and patient clinical complexity level, the variability of daily care demand in general hospitals directly impacts resource use. From the perspective of a single hospital, the extent of that impact depends on the degree of variation in care need, as well as on the hospital administrators' ability to adapt or otherwise respond to changes either in resource supply or demand. Our analysis on the demand dynamics of the Swiss health care system indicates that monitoring of care demand is useful to create surge capacity during disasters or the COVID-19 pandemic [20,47], by offering alternative solutions such as smoothing workloads and coordinating early discharges. It also has the potential to help health system planners and hospital managers to tailor their staffing and other resources to match care demand and the early planning of admissions (eg, surgeries or follow-up treatment) [48], to control patient flow for smoother service use.

What this analysis cannot describe is the human resources and other resources needed to meet care demands (ie, balancing care demands or any other supply-demand chain). To do so would require a full exploration of the relevant human resources (eg, physician and nurse staffing), in the light of each hospital's care demand. Furthermore, application of time-driven activity-based costing methods could provide a framework to identify process improvements for health care delivery $[49,50]$. However, we were not able to consider time-driven activity-based costing, as we do not have sufficient data regarding resource consumption (eg, personnel, equipment, and supplies) during the patients' journey along the clinical pathway [49].

Some studies have linked higher capacity utilization and patient turnover with adverse patient outcomes [5,21]. In addition to these results, acknowledging the effect of clinical complexity alongside capacity utilization and patient turnover might bring us closer to understanding the factors that stress hospital systems and the effects that a stressed system have on patient outcomes such as in-hospital mortality. Describing daily care demand to identify meaningful variation will require further studies (eg, examining patients' time-varying exposure to hospitals or units under pressure and the impact on the quality-of-care indicators and patient outcomes in causal models). Particularly, extending previous research on capacity utilization and in-hospital mortality [5,51] and using daily capacity utilization as time-varying exposure (ie, systemic stress factor), it would be of interest to explore the daily patient turnover and patient clinical complexity level as time-varying confounders. In a practical sense, this might also allow monitoring of pressure zones (eg, to manage care demand, where possible) in hospitals, which could reduce avoidable adverse events or death [5].

\section{Strengths and Limitations}

To our knowledge, this was the first study to explore hospital care demand dynamics via daily measurements of capacity utilization, patient turnover, and patient clinical complexity level on a national health system level. Furthermore, applying the standard methodology, programming, and software for large datasets allowed a longitudinal perspective by computing and visualizing demand dynamics per day of the year, day of the week, and weekdays vs weekends.

This study also had notable limitations. While we explored demand dynamics in detail, we could not do so with supply dynamics (eg, staffing, resources, etc) — an entire category of critical information in the demand-supply equation. Due to the large sample and FSO data composition (ie, aggregated data), it was also not possible to explore demand dynamics at the unit level - the interface between patients' care demand and health professionals' provision of care. Also, as we used codes assigned in routine data, the patient diagnoses and other variables could be biased by factors such as the accuracy of physicians' and nurses' documentation, lack of availability of ICD-10 codes for patients who were not discharged, and intentional upcoding of diagnoses to more expensive Swiss DRG categories [52,53]. 


\section{Conclusions}

This study illustrates daily care demand based on capacity utilization, patient turnover, patient clinical complexity level, and the variability of these factors between the 5 classes of Swiss general hospitals. For all 5 types, our analyses indicated distinct differences in capacity utilization, patient turnover, and patient clinical complexity level between days of the week, weekdays vs weekends, and seasons. This longitudinal study is a step toward detecting possible variables to be considered for time-varying exposure (eg, capacity utilization) and confounders (eg, patient clinical complexity level) in developing a casual model of tipping points and their links with quality of care or patient outcomes. Essentially, the variability of care demand provides a new perspective for gauging when hospitals are under strain, and this could help avoid pressure zones with a combination of appropriate resource allocation and care-demand planning in general hospitals.

\section{Acknowledgments}

We would like to thank the Swiss Federal Statistics Office (FSO) and its data management team for their support in providing inpatient data for our analyses. We are grateful to all the Swiss hospitals for their regular data reporting and information forwarded to the FSO. We would like to thank Mr Chris Shultis for English editing of this paper. We are also thankful to the Swiss Nurses' Association (SBK) for their financial support of this study. The funders had no role in the design, conduct, analysis, or report of this study.

\section{Authors' Contributions}

NS and MS had full access to all the data in the study and take responsibility for the integrity of the data and accuracy of the analysis. NS, RS, OE, DA, and MS contributed to the conception and design of the study. NS analyzed and drafted the manuscript. All the authors contributed to data interpretation and critically revised and edited the manuscript. All authors approved the final version.

\section{Conflicts of Interest}

None declared.

\section{Multimedia Appendix 1}

Description of variables and general characteristics of the study population for 5 Swiss general hospital types. [PDF File (Adobe PDF File), 87 KB-Multimedia Appendix 1]

\section{Multimedia Appendix 2}

Percentage of patient turnover by days of the year, days of the week (Monday to Sunday), and weekdays vs weekends over 1 year for 5 Swiss general hospital types.

[PDF File (Adobe PDF File), 498 KB-Multimedia Appendix 2]

\section{Multimedia Appendix 3}

Average patient clinical complexity levels by days of the year, days of the week (Monday to Sunday), and weekdays vs weekends over 1 year for 5 Swiss general hospital types.

[PDF File (Adobe PDF File), 405 KB-Multimedia Appendix 3]

\section{Multimedia Appendix 4}

Distribution of patient clinical complexity levels for the last 10 weeks of the year with the length of stay and average inpatients on weekly basis (showing complex patient stays over Christmas and the end of the year).

[PDF File (Adobe PDF File), 227 KB-Multimedia Appendix 4]

\section{References}

1. Relman A. Book Review Crossing the Quality Chasm: A New Health System for the 21st Century By the Committee on Quality of Health Care in America of the Institute of Medicine. 337 pp. Washington, D.C., National Academy Press, 2001. \$44.95. 0-309-07280-8. N Engl J Med 2001 Aug 30;345(9):702-703. [doi: 10.1056/nejm200108303450917] [Medline: $\underline{25057539]}$

2. Weber T. Optimal control theory with applications in economics. London: The MIT Press; 2011.

3. Belciug S, Gorunescu F. Improving hospital bed occupancy and resource utilization through queuing modeling and evolutionary computation. J Biomed Inform 2015 Feb;53:261-269 [FREE Full text] [doi: 10.1016/j.jbi.2014.11.010] [Medline: 25433363] 
4. Griffiths P, Saville C, Ball J, Jones J, Pattison N, Monks T, Safer Nursing Care Study Group. Nursing workload, nurse staffing methodologies and tools: A systematic scoping review and discussion. Int J Nurs Stud 2020 Mar;103:103487 [FREE Full text] [doi: 10.1016/j.ijnurstu.2019.103487] [Medline: $\underline{31884330}$ ]

5. Kuntz L, Mennicken R, Scholtes S. Stress on the Ward: Evidence of Safety Tipping Points in Hospitals. Management Science 2015 Apr;61(4):754-771. [doi: 10.1287/mnsc.2014.1917]

6. Freedman S. Capacity and Utilization in Health Care: The Effect of Empty Beds on Neonatal Intensive Care Admission. Am Econ J Econ Policy 2016 May 01;8(2):154-185 [FREE Full text] [doi: 10.1257/pol.20120393] [Medline: 27942353]

7. Sloan F. Hsieh C-R. Health economics. London: MIT Press; 2017.

8. Jones RP. Condition-specific growth in occupied beds in England following a sudden and unexpected increase in deaths. British Journal of Healthcare Management 2019 Jun 02;25(6):1-8. [doi: 10.12968/bjhc.2018.0026]

9. Friebel R, Fisher R, Deeny SR, Gardner T, Molloy A, Steventon A. The implications of high bed occupancy rates on readmission rates in England: A longitudinal study. Health Policy 2019 Aug;123(8):765-772 [FREE Full text] [doi: 10.1016/j.healthpol.2019.06.006] [Medline: 31262535]

10. Reader TW, Gillespie A. Patient neglect in healthcare institutions: a systematic review and conceptual model. BMC Health Serv Res 2013 Apr 30;13:156 [FREE Full text] [doi: 10.1186/1472-6963-13-156] [Medline: 23631468]

11. Ball JE, Murrells T, Rafferty AM, Morrow E, Griffiths P. 'Care left undone' during nursing shifts: associations with workload and perceived quality of care. BMJ Qual Saf 2014 Feb;23(2):116-125 [FREE Full text] [doi: 10.1136/bmjqs-2012-001767] [Medline: 23898215]

12. Wagner J, Gabler NB, Ratcliffe SJ, Brown SES, Strom BL, Halpern SD. Outcomes among patients discharged from busy intensive care units. Ann Intern Med 2013 Oct 01;159(7):447-455 [FREE Full text] [doi: 10.7326/0003-4819-159-7-201310010-00004] [Medline: 24081285]

13. Panagioti M, Khan K, Keers RN, Abuzour A, Phipps D, Kontopantelis E, et al. Prevalence, severity, and nature of preventable patient harm across medical care settings: systematic review and meta-analysis. BMJ 2019 Jul 17;366:14185 [FREE Full text] [doi: $10.1136 / \mathrm{bmj} .14185]$ [Medline: $\underline{31315828}$ ]

14. OECD. Health at a Glance 2017. OECD Publishing. 2017. URL: https://www.health.gov.il/publicationsfiles/ healthataglance2017.pdf [accessed 2020-07-08]

15. Fisher ES, Wennberg JE, Stukel TA, Skinner JS, Sharp SM, Freeman JL, et al. Associations among hospital capacity, utilization, and mortality of US Medicare beneficiaries, controlling for sociodemographic factors. Health Serv Res 2000 Feb;34(6):1351-1362 [FREE Full text] [Medline: 10654835]

16. Bed occupancy rate (\%), acute care hospitals only. Published. 2018. URL: https://gateway.euro.who.int/en/indicators/ hfa 542-6210-bed-occupancy-rate-acute-care-hospitals-only/ [accessed 2020-05-08]

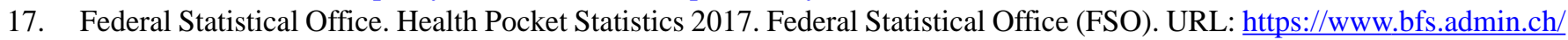
bfs/en/home/statistics/catalogues-databases/publications.assetdetail.4342090.html [accessed 2020-10-02]

18. Bujold M, Pluye P, Légaré F, Haggerty J, Gore GC, Sherif RE, Participatory Review Team. Decisional needs assessment of patients with complex care needs in primary care: a participatory systematic mixed studies review protocol. BMJ Open 2017 Nov 12;7(11):e016400 [FREE Full text] [doi: 10.1136/bmjopen-2017-016400] [Medline: 29133314]

19. VanFosson CA, Yoder LH, Jones TL. Patient Turnover: A Concept Analysis. ANS Adv Nurs Sci 2017;40(3):298-310. [doi: 10.1097/ANS.0000000000000171] [Medline: 28266962]

20. Chopra V, Toner E, Waldhorn R, Washer L. How Should U.S. Hospitals Prepare for Coronavirus Disease 2019 (COVID-19)? Ann Intern Med 2020 May 05;172(9):621-622 [FREE Full text] [doi: 10.7326/M20-0907] [Medline: 32160273]

21. Park SH, Blegen MA, Spetz J, Chapman SA, De Groot H. Patient turnover and the relationship between nurse staffing and patient outcomes. Res Nurs Health 2012 Jun;35(3):277-288. [doi: 10.1002/nur.21474] [Medline: 22457013]

22. Mendez CM, Harrington DW, Christenson P, Spellberg B. Impact of hospital variables on case mix index as a marker of disease severity. Popul Health Manag 2014 Feb;17(1):28-34 [FREE Full text] [doi: 10.1089/pop.2013.0002] [Medline: 23965045]

23. Menendez ME, Neuhaus V, van Dijk CN, Ring D. The Elixhauser comorbidity method outperforms the Charlson index in predicting inpatient death after orthopaedic surgery. Clin Orthop Relat Res 2014 Sep;472(9):2878-2886 [FREE Full text] [doi: 10.1007/s11999-014-3686-7] [Medline: 24867450]

24. Sharma N, Schwendimann R, Endrich O, Ausserhofer D, Simon M. Comparing Charlson and Elixhauser comorbidity indices with different weightings to predict in-hospital mortality: an analysis of national inpatient data. BMC Health Serv Res 2021 Jan 06;21(1):13 [FREE Full text] [doi: 10.1186/s12913-020-05999-5] [Medline: 33407455]

25. Quentin W, Geissler A, Busse R. Measuring and comparing health system outputs: using patient classification systems for efficiency analyses. In: Health system efficiency: European Observatory on Health Systems and Policies; 2016.

26. SwissDRG. Swiss Diagnosis Related Groups Version 8.0. Bern, Switzerland: SwissDRG AG; 2018. URL: https://www. swissdrg.org/de/akutsomatik/swissdrg [accessed 2020-12-05]

27. Bauer M, Ostermann H. DRGs in Transfusion Medicine and Hemotherapy in Germany. Transfus Med Hemother 2012 Apr;39(2):60-66 [FREE Full text] [doi: 10.1159/000337337] [Medline: 22670123]

28. Salisbury C, Boerma W. Balancing demand and supply in out-of-hours care. Florida: CRC Press; 2018. 
29. Fieldston E, Ragavan M, Jayaraman B, Metlay J, Pati S. Traditional measures of hospital utilization may not accurately reflect dynamic patient demand: findings from a children's hospital. Hosp Pediatr 2012 Jan;2(1):10-18. [doi: 10.1542/hpeds.2011-0016-2] [Medline: 24319808]

30. Macy ML, Stanley RM, Lozon MM, Sasson C, Gebremariam A, Davis MM. Trends in high-turnover stays among children hospitalized in the United States, 1993-2003. Pediatrics 2009 Mar;123(3):996-1002 [FREE Full text] [doi: 10.1542/peds.2008-1428] [Medline: 19255031]

31. Unruh LY, Fottler MD. Patient turnover and nursing staff adequacy. Health Serv Res 2006 Apr;41(2):599-612 [FREE Full text] [doi: $10.1111 / \mathrm{j} .1475-6773.2005 .00496 . x]$ [Medline: 16584467]

32. Norrish BR, Rundall TG. Hospital restructuring and the work of registered nurses. Milbank Q 2001;79(1):55-79; 2 p preceding VI [FREE Full text] [doi: 10.1111/1468-0009.00196] [Medline: 11286096]

33. DeLia D. Annual bed statistics give a misleading picture of hospital surge capacity. Ann Emerg Med 2006 Oct;48(4):384-8, 388.e1. [doi: 10.1016/j.annemergmed.2006.01.024] [Medline: 16997673]

34. De Pietro C, Camenzind P, Sturny I, Crivelli L, Edwards-Garavoglia S, Spranger A, et al. Switzerland: Health System Review. Health Syst Transit 2015;17(4):1-288, xix [FREE Full text] [Medline: 26766626]

35. Antoch J. Environment for statistical computing. Computer Science Review 2008 Aug;2(2):113-122. [doi: 10.1016/j.cosrev.2008.05.002]

36. Wickham H, Francois R. dplyr: A Grammar of Data Manipulation. 2020. URL: https://cran.r-project.org/web/packages/ dplyr/index.html [accessed 2020-08-28]

37. Wickham H. tidyr: Tidy Messy Data. 2020. URL: https://cran.r-project.org/web/packages/tidyr/index.html [accessed 2020-08-29]

38. Mailund T. Working with Dates: lubridate. New York: Springer; 2019:195-203.

39. Wickham H. stringr: Simple, Consistent Wrappers for Common String Operations. stringr: Simple, Consistent Wrappers for Common String Operations. 2019. URL: https://cran.rstudio.com/web/packages/stringr/index.html [accessed 2020-02-10]

40. Wickham H. ggplot2legant graphics for data analysis. New York: Springer; 2016.

41. Pedersen T. patchwork: The Composer of Plots. 2020. URL: https://cran.r-project.org/web/packages/patchwork/index.html [accessed 2020-12-18]

42. Wickham H. scales: Scale Functions for Visualization. 2020. URL: https://cran.r-project.org/web/packages/scales/index. html [accessed 2020-05-25]

43. Park SH, Dunton N, Blegen MA. Comparison of Unit-Level Patient Turnover Measures in Acute Care Hospital Settings. Res Nurs Health 2016 Jun;39(3):197-203. [doi: 10.1002/nur.21715] [Medline: 26998744]

44. SwissDRG. SwissDRG System 6. 2017. URL: https://www.swissdrg.org/de/akutsomatik/archiv-swissdrg-system/ swissdrg-system-602017 [accessed 2020-05-04]

45. Antioch K, Zhang X. Organ transplant AN-DRGs: modifying the exceptions hierarchy in casemix classification. Aust Health Rev 2000;23(1):137-152. [doi: 10.1071/ah000137] [Medline: 10947598]

46. Pauls LA, Johnson-Paben R, McGready J, Murphy JD, Pronovost PJ, Wu CL. The Weekend Effect in Hospitalized Patients: A Meta-Analysis. J Hosp Med 2017 Sep;12(9):760-766. [doi: 10.12788/jhm.2815] [Medline: 28914284]

47. Mills AF, Helm JE, Wang Y. Surge Capacity Deployment in Hospitals: Effectiveness of Response and Mitigation Strategies. M\&SOM 2020 Mar 30:367-387. [doi: 10.1287/msom.2019.0838]

48. Shabanikiya H, Gorgi HA, Seyedin H, Jafari M. Assessment of Hospital Management and Surge Capacity in Disasters. Trauma Mon 2016 May;21(2):e30277 [FREE Full text] [doi: 10.5812/traumamon.30277] [Medline: 27626015]

49. Martin JA, Mayhew CR, Morris AJ, Bader AM, Tsai MH, Urman RD. Using Time-Driven Activity-Based Costing as a Key Component of the Value Platform: A Pilot Analysis of Colonoscopy, Aortic Valve Replacement and Carpal Tunnel Release Procedures. J Clin Med Res 2018 Apr;10(4):314-320 [FREE Full text] [doi: 10.14740/jocmr3350w] [Medline: 29511420]

50. Keel G, Savage C, Rafiq M, Mazzocato P. Time-driven activity-based costing in health care: A systematic review of the literature. Health Policy 2017 Jul;121(7):755-763 [FREE Full text] [doi: 10.1016/j.healthpol.2017.04.013] [Medline: 28535996]

51. Madsen F, Ladelund S, Linneberg A. High levels of bed occupancy associated with increased inpatient and thirty-day hospital mortality in Denmark. Health Aff (Millwood) 2014 Jul;33(7):1236-1244. [doi: 10.1377/hlthaff.2013.1303] [Medline: 25006151]

52. Quan H, Eastwood C, Cunningham CT, Liu M, Flemons W, De Coster C, IMECCHI investigators. Validity of AHRQ patient safety indicators derived from ICD-10 hospital discharge abstract data (chart review study). BMJ Open 2013 Oct 10;3(10):e003716 [FREE Full text] [doi: 10.1136/bmjopen-2013-003716] [Medline: 24114372]

53. Wasserfallen J, Zufferey J. Financial impact of introducing the Swiss-DRG reimbursement system on potentially avoidable readmissions at a university hospital. Swiss Med Wkly 2015;145:w14097 [FREE Full text] [doi: 10.4414/smw.2015.14097] [Medline: 25659119] 


\section{Abbreviations}

DRG: diagnostic-related group

FSO: Federal Statistics Office

ICD: International Classification of Diseases

LOS: length of stay

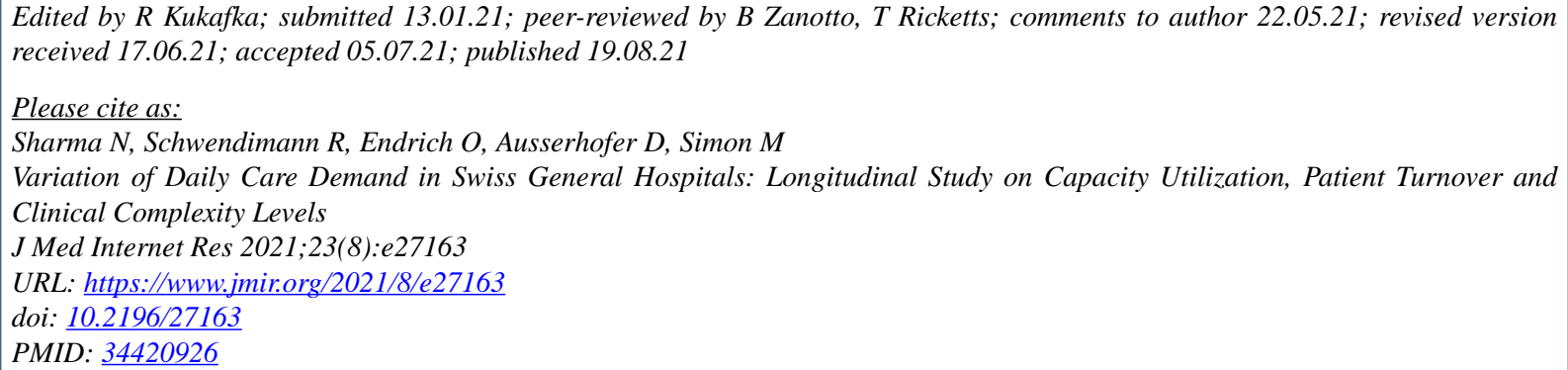

CNarayan Sharma, René Schwendimann, Olga Endrich, Dietmar Ausserhofer, Michael Simon. Originally published in the Journal of Medical Internet Research (https://www.jmir.org), 19.08.2021. This is an open-access article distributed under the terms of the Creative Commons Attribution License (https://creativecommons.org/licenses/by/4.0/), which permits unrestricted use, distribution, and reproduction in any medium, provided the original work, first published in the Journal of Medical Internet Research, is properly cited. The complete bibliographic information, a link to the original publication on https://www.jmir.org/, as well as this copyright and license information must be included. 